\title{
Comparison between Laparoscopic Ultrasound and Intraoperative Cholangiogram in Detection of Common Bile Duct Stones during Laparoscopic Cholecystectomy for Cholelithiasis: A Prospective Study
}

\author{
Mohamed Elkerkary ${ }^{1} \odot$, Gouda Ellabban², Mohamed Shams ${ }^{3}$, Mostafa Abdel-Raheem ${ }^{4}$, Hamdy Shaban ${ }^{5}$, Ahmed Hassan ${ }^{6}$
}

\begin{abstract}
Introduction: Intraoperative cholangiography (IOC) during laparoscopic cholecystectomy (LC) is valuable in the detection of biliary abnormalities. In this study, we aimed to investigate the diagnostic accuracy of IOC during LC for the detection of anatomic variations of the biliary system, as well as the visualization ability of IOC on determining the normal anatomy of the biliary tree.

Materials and methods: This cross-sectional study was conducted on patients who were presented to the surgery outpatient clinic and were scheduled for elective LC for symptomatic cholelithiasis. Patients underwent intraoperative laparoscopic ultrasound (LUS) before the dissection of Calot's triangle and IOC video fluoroscopy examination of the extrahepatic biliary tree.

Results: Our study enrolled 53 patients. No intraoperative complications occurred in all enrolled patients. LUS was successful in all 53 (100\%) cases, while IOC was successful in 50 (94.3\%) cases. IOC had accuracy rate of 100\% (50 patients) in defining biliary ducts at the porta hepatis compared to $84.91 \%$ (45 patients) for LUS with a failure rate of $15.09 \%(p=0.60)$. Concerning stones detection, LUS accuracy indexes were as follows: sensitivity $=80 \%$; specificity $=95.83 \%$; positive predictive value $(\mathrm{PPV})=66.67 \%$; negative predictive value (NPV) $=97.87 \%$ 99; and diagnostic odds ratio $(D O R)=92$. IOC accuracy indexes were as follows: sensitivity $=80 \%$; specificity $=93.33 \% ; P P V=57.14 \% ; N P V=90 \%$; and DOR $=56$. Conclusion: The results of the current study encourage using IOC as an effective, accurate, feasible, and safe technique to visualize the biliary tree while performing LC.

Keywords: Diagnostic accuracy, Intraoperative cholangiography, Laparoscopic cholecystectomy.

World Journal of Laparoscopic Surgery (2021): 10.5005/jp-journals-10033-1449
\end{abstract}

\section{INTRODUCTION}

Throughout recent decades, minimally invasive surgery in several abdominal procedures has been growing. Laparoscopic procedures are the most commonly performed procedures in the last few years. ${ }^{1}$ The surgeon's failure to palpate abdominal organs is a downside of this laparoscopic technique. However, laparoscopic cholecystectomy (LC) is an easy and safe procedure as long as there is a clear mapping of the biliary duct. ${ }^{2,3}$ Nonetheless, the description and detailed evaluation of biliary pathways are critical for the identification and prevention of the bile duct injury (BDI) of the common bile duct stones (CBDS). ${ }^{4,5}$ The invisibility of the biliary tract is the main cause of BDI in $97 \%$ of diagnosed cases. ${ }^{6,7}$ Moreover, the inadequate skills of surgeons may participate in BDI. To avoid this serious complication that may affect the outcome of the procedure and the quality of life, different techniques have been proposed such as intraoperative cholangiography (IOC), passive infrared cholangiography, dye cholangiography, and laparoscopic ultrasound (LUS). ${ }^{4,8,9}$

Regarding IOC, there is a large debate as to whether IOC should be used routinely or for select cases. ${ }^{10}$ However, it is the most commonly used technique to determine the biliary duct. ${ }^{11}$ Some limitations were reported for the use of IOC, such as prolongation of the procedure time, cost, and the presence of the risk of an inflamed cystic duct and ionizing radiation..$^{12,13}$ LUS is a less invasive, cheaper, and faster technique when compared with IOC. ${ }^{14}$ A recent meta-analysis showed that the sensitivity and
1-3,5,6 Department of Surgery, Faculty of Medicine, Suez Canal University, Ismailia, Egypt

${ }^{4}$ Department of Surgery, Hywel Dda University Health Board, Wales, United Kingdom

Corresponding Author: Mohamed Elkerkary, Department of Surgery, Faculty of Medicine, Suez Canal University, Ismailia, Egypt, e-mail: m_kerkary@med.suez.edu.eg

How to cite this article: Elkerkary M, Ellabban G, Shams M, et al. Comparison between Laparoscopic Ultrasound and Intraoperative Cholangiogram in Detection of Common Bile Duct Stones during Laparoscopic Cholecystectomy for Cholelithiasis: A Prospective Study. World J Lap Surg 2021;14(2):69-74.

Source of support: Nil

Conflict of interest: None

specificity of IOC and LUC were comparable. However, LUC was associated with lower ionizing radiation, lower failure rate, and much quicker performance and can be repeated safely during the procedure. ${ }^{15}$ The success rate of LUC is estimated to be $100 \%$ compared to $91.3 \%$ for IOC. Moreover, some reports demonstrated that the sensitivity of IOC combined with LUS was greater than that of IOC and LUS took separately. ${ }^{8,16-18}$ Therefore, this study aimed to investigate the diagnostic accuracy of $\mathrm{IOC}$ in combination with LUC during LC. 


\section{Materials and Methods}

\section{Study Population}

This cross-sectional study was conducted at the Surgery Department of Suez Canal University Hospital, Ismailia, Egypt. It was performed on patients who were presented to the surgery outpatient clinic and were scheduled for elective LC for symptomatic cholelithiasis. This study included patients who were scheduled for LC for symptomatic cholelithiasis and were stratified as low-risk of having CBD stones. The patients who fulfilled the inclusion criteria were allocated to a sampling frame and randomized by simple random sampling. Ethical approval was obtained from the Surgery Department at Suez Canal University Hospital. Patients were notified about the study, and the informed written consent was obtained prior to participation in the study.

We included patients with symptomatic cholelithiasis between the ages of 18 years (for easy laparoscopic instrumentation with LUS $10-\mathrm{mm}$ probe) and 65 years (more comorbidities as a relative contraindication to $L C$ ). We excluded patients with contraindications to LC, complicated cholelithiasis (e.g. obstructive jaundice and acute pancreatitis), previous gastrointestinal surgery, contrast hypersensitivity, previous endoscopic retrograde cholangiopancreatography (ERCP), and CBD stent due to radiological falsies, or conversion to open cholecystectomy during LC.

\section{Data Collection}

All enrolled patients were subjected to history taking for exclusion criteria and comorbidities, clinical examination for signs of cholelithiasis, laboratory investigations (liver functions and coagulation profile), and a recent transabdominal US examination that includes sizes of the gallbladder and CBD, wall thickness, presence of stones, masses, polyps, or fluid around the gallbladder, as well as the status of the pancreatic head. Patients then underwent intraoperative LUS before dissection of Calot's triangle and IOC video fluoroscopy examination of the extrahepatic biliary tree.

\section{Laparoscopic Ultrasound}

We introduced the deflectable multifrequency $(7.5-10 \mathrm{MHz})$ endosonography linear probe through a $10-\mathrm{mm}$ port, while the camera was placed through the midepigastric port. First, the liver was scanned and the CBD was identified. The gallbladder and liver were retracted superiorly and cephalad. Sometimes the junction of the right and left hepatic ducts could be seen. The CBD was followed to the duodenum. A transverse view of the bile duct could be obtained by acute deflection of the transducer.

\section{Intraoperative Cholangiography}

We initially dissected the Calot's triangle to identify the cystic duct and artery, which was divided between clips. To apply the cholangiocatheter, we dissected the cystic duct free for about $3 \mathrm{~cm}$ and then applied a ligature on the junction of the $G B$ and the cystic duct. The cholangiocatheter was introduced through the midclavicular port or through a separate puncture in the right upper quadrant. Utilizing dynamic fluoroscopy, we obtained a scout film to localize the tip of the cholangiocatheter. First, only 2 to 3 cc of a water-soluble contrast dye with $25 \%$ concentration (diatrizoic acid: Gastrografin and sometimes Omnipaque) were injected identifying the cystic duct-CBD junction. The fluoroscopy unit was shifted caudally a few centimeters, and the course of the distal CBD was identified by injecting another 5 cc of contrast. The fluoroscopy arm was shifted cephalad, and another 5 cc of contrast is injected to visualize the common hepatic duct and the proximal hepatic radicals. When the cholangiogram was done, the clamp and catheter were removed and two clips were placed just distal to the ductotomy.

\section{Statistical Analysis}

The formula for the sample size was as follows: $n$ (per test $)=[a / 2+\beta / 2]^{2} *\left[\left(p_{1} *\left(1-p_{1}\right)\right)+\left(p_{2} *\left(1-p_{2}\right)\right)\right] /\left[p_{1}-p_{2}\right]^{2}$ where $n=$ the sample size required in each group, $p_{1}=$ sensitivity of LUS in choledocholithiasis $=96 \%, p_{2}=$ sensitivity of IOC in choledocholithiasis $=75 \%$, $a$ depends on desired significance level $(95 \%)=1.96$, and $\beta$ depends on desired power $(90 \%)=1.28$. Thus, the sample included 53 patients who fulfilled the inclusion criteria. A data entry form was created using Epi Info 7.0, and the same software was utilized for statistical analysis along with the SPSS 16 for advanced statistics. Continuous data were expressed as mean and stander deviation, and categorical data were expressed as frequencies and percentages. Continuous data with normal distribution were compared using the Student's $t$-test or ANOVA, while the Mann-Whitney/Wilcoxon two-sample test was used to compare two-sample variables with other distributions. The accuracy indexes of LUS and IOC were expressed as sensitivity, specificity, positive predictive value (PPV), negative predictive value (NPV), and diagnostic odds ratio (DOR) with $95 \%$ confidence interval. The significance level was considered at $0.05 \times$.

\section{Results}

\section{Baseline Data}

Our study enrolled 53 patients: 17 males and 3 females. The mean ages for male and female groups were $41.35 \pm 8.48$ and $40.06 \pm 11.85$ years, respectively (age was statistically comparable between both genders; $p=0.69$ ). Of the patients, $22(41.5 \%)$ had multiple stones and 31 (58.5\%) had solitary stones on preoperative US.

\section{Intra- and Postoperative Complication Rates}

No intraoperative complications occurred in all enrolled patients. In terms of 30 days' follow-up, only nine (17\%) had postoperative complications that included chest infections (three), intraabdominal collection (two), urinary tract infection (one), and wound infections (three). No mortalities were recorded during the follow-up period.

\section{LUS vs IOC Success Rates}

LUS was successful in all $53(100 \%)$ cases, while IOC was successful in $50(94.3 \%)$ cases. Using the Chi-square test to compare the success rate between LUS and IOC, we observed no significant difference between both tests $(\mathrm{OR}=1.0061 ; p=0.08)$. The reasons for the three observed failures in IOC included narrow cystic duct, thick valves at cystic duct, and technical failure.

\section{Time to Complete the Procedure}

In terms of the time to complete the procedure, LUS took $12.53 \pm 2.56$ minutes to complete with a range of 6 to 17 minutes, while IOC took $8.66 \pm 2.77$ minutes to complete with a range of 7 to 15 minutes. Comparing both procedures using the Mann-Whitney/Wilcoxon Test showed a significantly longer 
time in LUS compared to IOC ( $p=0.001)$. We carried out a linear regression analysis to see if there was a correlation between LUS and $I O C$ regarding time to complete. We found that there was a positive correlation between LUS and IOC for time with Pearson's correlation coefficient of 0.4225 . This correlation was statistically significant ( $p$-value $<0.0016$ ) (Fig. 1).

The learning curve was longer for LUS than for IOC. LUS took a longer time to complete in the first 30 patients and then started to decline with Pearson's correlation coefficient of -0.8717 and $p$-value $<0.0001$ using linear regression analysis. IOC had a less steep learning curve with time to complete dropping by 20 patients and Pearson's correlation coefficient $=-0.4788$ and $p$-value $<0.0003$ using linear regression analysis (Fig. 2).

\section{Accuracy of LUS and IOC in Defining Biliary Tract Structures}

IOC had an accuracy rate of 100\% (50 patients) in defining biliary ducts at the porta hepatis compared to $84.91 \%$ (45 patients) for LUS with a failure rate of $15.09 \%$. Statistical analysis utilizing a $2 \times 2$ contingency

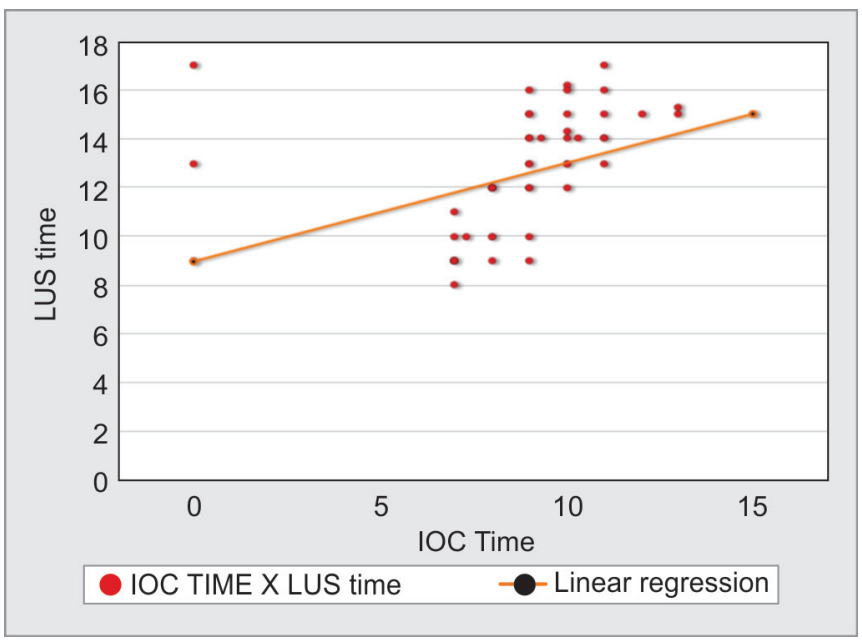

Fig. 1: Correlation between LUS time and IOC time. Pearson's correlation coefficient $=0.4225$. $p$-value $<0.0016$ using linear regression analysis table showed a nonsignificant difference between the two modalities $(p=0.60)$ (Fig. 3). Similar findings also occurred when evaluating the two modalities for the accuracy in defining extrahepatic biliary ducts (CBD, common hepatic duct (CHD), and cystic duct). LUS had a failure rate of $3.77 \%$ (2 patients) and a success rate of $96.23 \%$ (51 patients), while IOC was successful in $100 \%$ of cases (50 patients) in defining extrahepatic ducts. Statistical analysis utilizing a $2 \times 2$ contingency table showed a nonsignificant difference between the two modalities ( $p$-value $=0.88$ ) (Fig. 4).

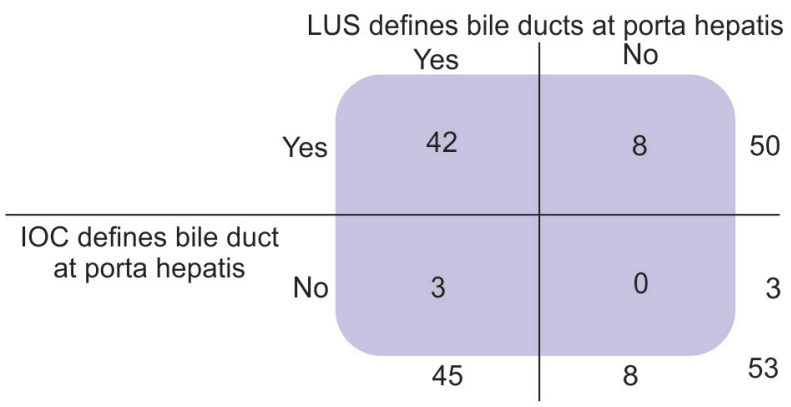

Fig. 3: A $2 \times 2$ contingency table for accuracy analysis between LUS and $I O C$ in defining bile ducts at porta hepatis. $X^{2}$ Fisher-exact one-tailed $p$-value $=0.60$

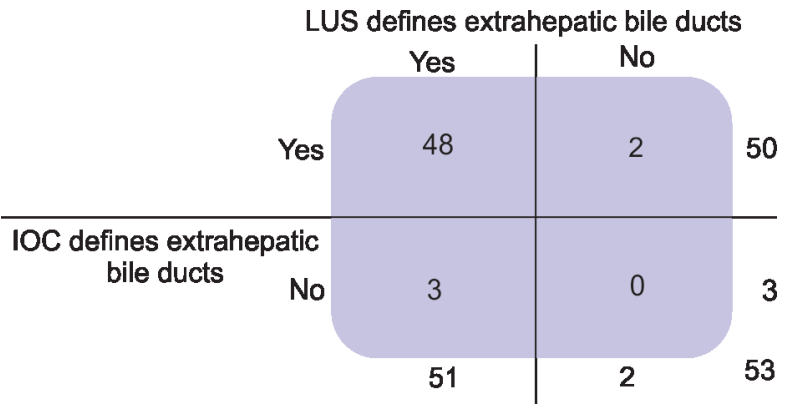

Fig. 4: A $2 \times 2$ contingency table for accuracy analysis between LUS and IOC in defining extrahepatic bile ducts. $X^{2}$ Fisher-exact one-tailed $p$-value $=0.88$
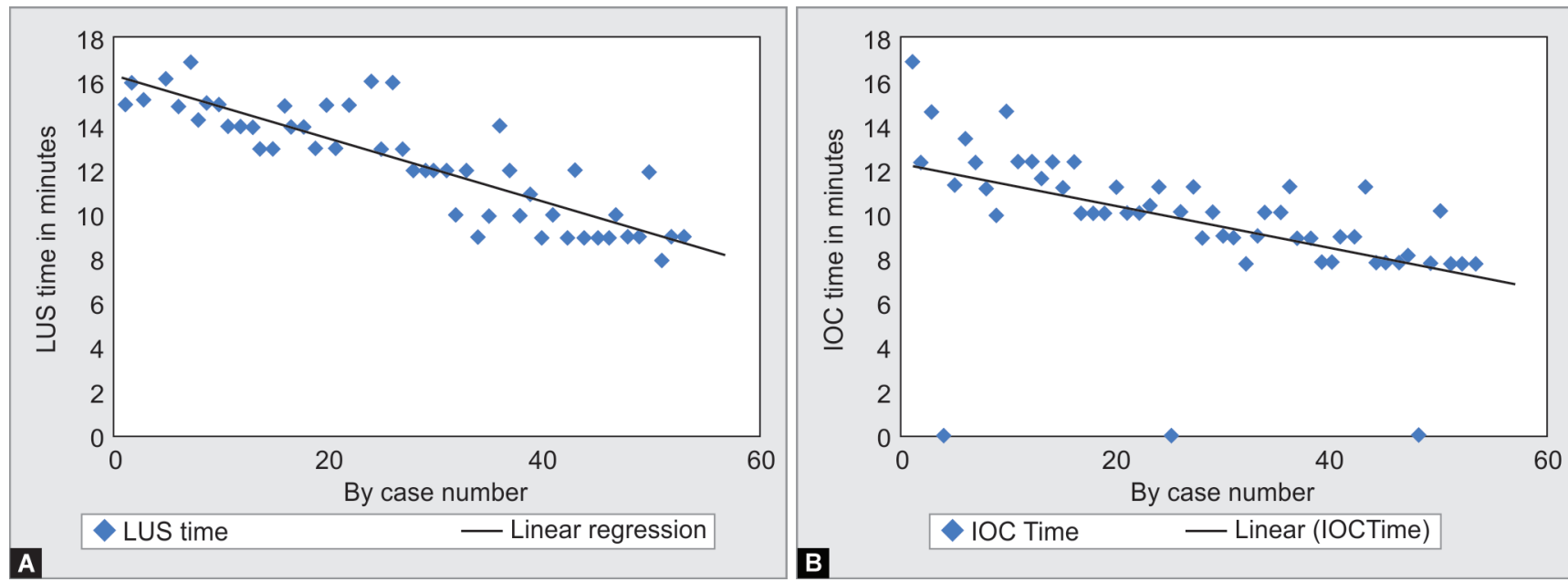

Figs 2A and B: (A) LUS learning curve by time in minutes. Pearson's correlation coefficient $=-0.8717$. $p$-value $<0.0001$ using linear regression analysis; (B) IOC learning curve by time in minutes. Pearson's correlation coefficient $=-0.4788$. $p$-value $<0.0003$ using linear regression analysis 
However, LUS failed to detect CD junction anomalies in all patients, while IOC detected these anomalies in 4 patients (8\%) out of 50 patients. The anomalies found were medial insertion of cystic duct in one patient (2\%) and low insertion of cystic duct in three patients $(6 \%)$. The incidence of these anomalies was statistically insignificant ( $p$-value $=0.05$ ). While LUS detected vascular structures in 52 patients (98.11\%) with an OR of 1.554, it failed to demonstrate anomalies in the vascular structures in all patients (Table 1).

\section{Postoperative CBD Stones}

Within the 6-month follow-up period, we suspected postoperative CBD stones in 7 patients $(13.2 \%)$ among the 53 total sample. Of those seven patients, one patient presented with biliary pancreatitis and was treated conservatively. Two patients had persistent elevation of LFTs. Three patients underwent magnetic resonance cholangiopancreatography (MRCP) postoperatively, who were both IOC and LUS positive for CBD stones and MRCP confirmed the presence of stones. One patient had CBD dilatation on transabdominal US, who also was LUS and IOC positive. These stones were detected after one (three stones), two (one stone), three (two stones), and 4 months (one stone) of follow-up.

All seven patients underwent ERCP (13.21\%). This number is quite high due to the fact that the selected sample was the lowrisk group for $\mathrm{CBD}$ stones. Of the seven patients, who underwent ERCP, five $(71.43 \%)$ showed CBD stones and were extracted; the finding was included as end point true positive, while in two patients $(28.57 \%)$, it failed to demonstrate any CBD stones and were included as end point true negatives. The true incidence of concomitant CBD stones in our series was $9.43 \%$ by ERCP.

\section{Accuracy of LUS and IOC in Detecting CBD Stones}

LUS was true positive in 4 patients $(7.55 \%)$, false positive in 2 patients $(3.77 \%)$, false negative in 1 patient $(2 \%)$, and true negative in 46 patients $(86.67 \%)$. LUS accuracy indexes were as follow: sensitivity $=80 \%(95 \% \mathrm{Cl} 0.29-0.98)$; specificity $=95.83 \%(95 \% \mathrm{Cl}$ $0.85-0.99) ; \mathrm{PPV}=66.67 \%(95 \% \mathrm{Cl} 0.24-0.94) ; \mathrm{NPV}=97.87 \%(95 \% \mathrm{Cl}$ 0.87-0.99); and DOR = 92 (95\% Cl 6.77-1249.72) (Table 2).

IOC was true positive in 4 patients $(8 \%)$, false positive in 3 patients $(6 \%)$, false negative in 1 patient $(2 \%)$, and true negative in 42 patients (84\%). IOC accuracy indexes were as follow:

Table 1: Accuracy of LUS and IOC in defining biliary tract structures

\begin{tabular}{llcc}
\hline Bile ducts at porta & Yes & $45(84.91 \%)$ & $50(100 \%)$ \\
hepatis & No & $8(15.09 \%)$ & $0(0 \%)$ \\
\multirow{2}{*}{ Extrahepatic bile ducts } & Yes & $51(96.23 \%)$ & $50(100 \%)$ \\
& No & $2(3.77 \%)$ & $0(0 \%)$ \\
\multirow{2}{*}{ Biliary ductal anomalies } & Yes & $0(0 \%)$ & $4(8 \%)$ \\
& No & $53(100 \%)$ & $46(92 \%)$ \\
Vascular structures & Yes & $52(98.11 \%)$ & $0(0 \%)$ \\
& No & $1(1.89 \%)$ & $53(100 \%)$ \\
\hline
\end{tabular}

Table 2: LUS accuracy indexes

\begin{tabular}{llcc}
\hline LUS & $\begin{array}{l}\text { CBD stone }(s) \\
\text { present }\end{array}$ & $\begin{array}{l}\text { CBD stone }(s) \text { not } \\
\text { present }\end{array}$ & \multicolumn{1}{c}{ Total } \\
\hline Positive & $4(7.55 \%)$ & $2(3.77 \%)$ & $6(11.32 \%)$ \\
Negative & $1(2 \%)$ & $46(86.68 \%)$ & $47(88.68 \%)$ \\
Total & $5(9.55 \%)$ & $48(90.45 \%)$ & $53(100 \%)$ \\
\hline
\end{tabular}

Table 3: $I O C$ accuracy indexes

\begin{tabular}{llcc}
\hline & CBD stone $(s)$ & CBD stone $(s)$ not & Total \\
IOC & present N(\%) & present N (\%) & $N(\%)$ \\
\hline Positive & $4(8 \%)$ & $3(6 \%)$ & $7(14 \%)$ \\
Negative & $1(2 \%)$ & $42(84 \%)$ & $43(86 \%)$ \\
Total & $5(10 \%)$ & $45(90 \%)$ & $50(100 \%)$ \\
\hline
\end{tabular}

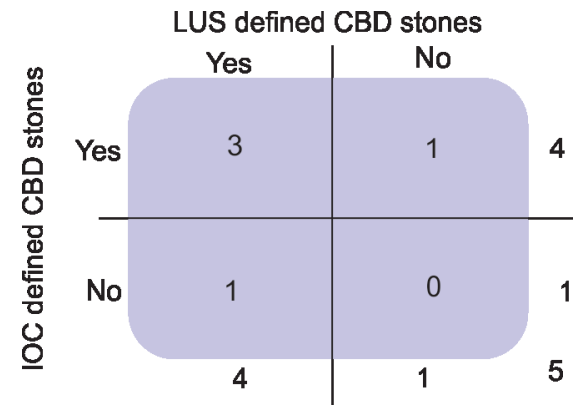

Fig. 5: A $2 \times 2$ contingency table for accuracy analysis between LUS and IOC in defining CBD stones. $X^{2}$ Fisher-exact one-tailed $p$-value $=0.8$ after stratification by true-positive results of $C B D$ stones presence

sensitivity $=80 \%(95 \% \mathrm{Cl} 0.29-0.98) ;$ specificity $=93.33 \% \%(95 \%$ $\mathrm{Cl} 0.81-0.98) ; \mathrm{PPV}=57.14 \%(95 \% \mathrm{Cl} 0.20-0.88) ; \mathrm{NPV}=90 \%(95 \% \mathrm{Cl}$ 0.86-0.99); and DOR $=56$ (95\% Cl 4.67-671.89) (Table 3).

A $2 \times 2$ contingency table for accuracy analysis between LUS and IOC in defining CBD stone was constructed after stratification and adjustment by ERCP end point true-positive results. The $p$-value was 0.8 that showed no significant difference between the two modalities in detection of CBD stones during $L C$ regarding their accuracy indexes. The analysis was carried out and showed equivalence/noninferiority between both tests by using Fisherexact test for $X^{2}$ (Fig. 5).

\section{Discussion}

CBD imaging during cholecystectomy has been an issue of debate for decades; some surgeons will routinely image the CBD for all cholecystectomy cases and others will use it selectively based on preoperative indicators. ${ }^{19}$ The mainstay imaging modality was $I O C$, in which the biliary tree is cannulated and a contrast material will be injected through the biliary system with either spot films or dynamic fluoroscopy. ${ }^{20}$ The issue of the clinical relevance of this technique is at least questionable, and with the search of the literature, a definitive answer could not be found. LUS is another modality, which was introduced into clinical practice, but was never widely adopted. ${ }^{21}$ The data from the literature are promising, but no enough evidence could be found with few superiority or equality/ noninferiority studies.Both techniques are not regularly utilized in our institution as biliary imaging is carried out only by IOC in selected patients based on individualized patient criteria.

We performed a cross-sectional study of low-risk patients for CBD stones. Sample size calculation yielded 53 patients who underwent LC as planned with evaluation of the biliary tree by both LUS and IOC. We adopted an equality/noninferiority analysis to assess statistical significance because our data did not enable superiority analysis. Patients who demonstrated filling defects by both modalities were managed expectantly and followed up for 6 months to assess the clinically significant CBD stones; the end 
point was assurance of true-positive results, which was defined as CBD stone extraction by ERCP for the clinically manifested CBD stones if evident during follow-up.

LUS was successfully carried out in all 53 patients with a success rate of $100 \%$, while IOC was successful in 50 patients (94.34\%). The difference in the success rate between LUS and IOC was statistically insignificant $(p=0.08)$. These results correlates with the literature as the IOC success rate was reported to range from 83 to $100 \%$ by several studies, such as the systematic review by Ford and colleagues. ${ }^{22} \mathrm{IOC}$ failure was due to technical problem in the C-arm, narrow cystic duct that could not be cannulated and thick valves of Heister at the cystic duct totally obscuring the duct lumen.

IOC took less time to complete compared to LUS in our series, and this difference was statistically significant $(p=0.001)$. This can be explained in part by the fact that LUS takes a longer learning curve, ${ }^{23,24}$ and in this study, it was the first time to utilize this modality; in another part, the IOC requires less laparoscopic surgical skills with little familiarity with the technique. We carried out a linear regression analysis to see if there was a correlation between LUS and IOC regarding time to complete. We found a significant positive correlation between LUS and IOC time. The learning curve was longer for LUS than for IOC. LUS took a longer time to complete in the first 30 patients and then started to decline. IOC had less steep learning curve with time to complete dropping by 20 patients.

IOC had an accuracy rate of $100 \%$ in defining biliary ducts at the porta hepatis compared to $84.9 \%$ (45 patients) for LUS with a failure rate of $15.1 \%$. Data analysis showed a nonsignificant difference between the two modalities $(p=0.6)$. This failure rate can be explained by the difficulty in locating the US probe with good alignment to obtain images of biliary structures at the porta hepatis. Further, the imaging planes of LUS are totally different from the classical transverse and longitudinal planes of transabdominal US. This issue was raised by several authors and came to the same conclusion. ${ }^{25}$ Similar findings also occurred when evaluating the two modalities for the accuracy in defining extrahepatic biliary ducts (CBD, CHD, and cystic duct). The failure of LUS was mainly at the area of the distal CBD, where it was covered by the gases of the duodenum, and probably with the increase in the learning curve, this issue can be resolved. Several studies demonstrated similar results, making $\mathrm{IOC}$ the gold standard in defining biliary anatomy. ${ }^{25}$ While LUS detected vascular structures in 52 patients, it failed to demonstrate anomalies in the vascular structures in all patients. Owing to the wide variability of vascular anomalies in this region of the human body, the false-negative results have high probability. In the absence of a gold standard test to compare, these results cannot be ascertained.

LUS detected CBD stones in 6 out of 53 patients (11.32\%), while IOC detected CBD stones in 7 out of 50 patients (14\%). The difference in the rate of detection of CBD stones by the two modalities was statistically insignificant $(p=0.45)$. Several studies showed that the incidence of concomitant gallstones and CBD stones range from 11 to $21 \%$ at time of surgery. ${ }^{26,27}$ In our study, we wanted to assess the true-positive incidence of CBD stones and to evaluate the clinically significant proportion. All patients were followed up postoperatively for 6 months with expectant approach and were informed about the possibility of having CBD stones. They were informed about warning symptoms and instructed to return to the hospital if any occurred plus their follow-up appointments. During follow-up, seven patients (13.21\%) were suspected of having CBD stones. A former study reported that $\mathrm{CBD}$ stones may take up to 18 months from LC to manifest clinically. ${ }^{28}$
All seven patients underwent ERCP (13.2\%). This proportion was statistically insignificant $(p=0.8)$ due to the fact that the selected sample was the low-risk group for CBD stones. Of the seven patients who underwent ERCP, five showed CBD stones and were extracted, and the finding was included as end point true positive. The true incidence of concomitant CBD stones in our series was $9.43 \%$ by ERCP. Our results of accuracy indexes analysis of LUS and IOC correlate with the literature we reviewed regarding diagnosis of CBD stones. ${ }^{25}$

The study limitations include the relatively small sample size, which may have hindered the detection of significant differences between the two modalities. Further, one of the secondary objectives (the evaluation of the liver parenchyma using LUS) could not be investigated, which was due to time restrictions. Future studies should enroll a larger sample size and attempt to avoid the restrictions mentioned in our study. Further, longer-term follow-up may provide more data regarding this comparison.

In conclusion, our analysis showed noninferiority between IOC and LUS in terms of CBD stone detection; however, IOC had a higher ability to visualize the anatomy of the biliary tracts and vascular structures/anomalies.

\section{OrCID}

Mohamed Elkerkary @ https://orcid.org/0000-0002-3998-265X

\section{References}

1. Santambrogio R, Montorsi M, Bianchi $P$, et al. Common bile duct exploration and laparoscopic cholecystectomy: role of intraoperative ultrasonography. J Am Coll Surg 1997;185(1):40-48. DOI: 10.1016/ s1072-7515(97)00013-6.

2. Giger U, Michel JM, Vonlanthen R, et al. Laparoscopic cholecystectomy in acute cholecystitis: indication, technique, risk and outcome. Langenbeck's Arch Surg 2005;390(5):373-380. DOI: 10.1007/s00423004-0509-4.

3. Dubois F, Icard P, Berthelot G, et al. Coelioscopic cholecystectomy. Ann Surg 1990;211(1):60-62. DOI: 10.1097/00000658-19900100000010.

4. Pesce A, Piccolo G, La Greca G, et al. Utility of fluorescent cholangiography during laparoscopic cholecystectomy: a systematic review. World J Gastroenterol 2015;21(25):7877-7883. DOI: 10.3748/ wjg.v21.i25.7877.

5. Ding GQ, Cai W, Qin MF. Is intraoperative cholangiography necessary during laparoscopic cholecystectomy for cholelithiasis? World J Gastroenterol 2015;21(7):2147-2151. DOI: 10.3748/wjg.v21.i7.2147.

6. Connor S, Garden OJ. Bile duct injury in the era of laparoscopic cholecystectomy. Br J Surg 2006;93(2):158-168. DOI: 10.1002/bjs.5266.

7. Way LW, Stewart L, Gantert W, et al. Causes and prevention of laparoscopic bile duct injuries: analysis of 252 cases from a human factors and cognitive psychology perspective. Ann Surg 2003;237(4):460-469. DOI: 10.1097/01.SLA.0000060680.92690.E9.

8. Hublet A, Dili A, Lemaire J, et al. Laparoscopic ultrasonography as a good alternative to intraoperative cholangiography (IOC) during laparoscopic cholecystectomy: results of prospective study. Acta Chir Belg 109(3):312-316. DOI: 10.1080/00015458.2009.11680431.

9. Halawani HM, Tamim H, Khalifeh $\mathrm{F}$, et al. Impact of intraoperative cholangiography on postoperative morbidity and readmission: analysis of the NSQIP database. Surg Endosc 2016;30(12):5395-5403. DOI: 10.1007/s00464-016-4896-8.

10. Martin B, Ong EGP. Selective intraoperative cholangiography during laparoscopic cholecystectomy in children is justified. J Pediatr Surg 2018;53(2):270-273. DOI: 10.1016/j.jpedsurg.2017.11.017.

11. Giulea C, Enciu O, Bîrcă T, et al. Selective intraoperative cholangiography in laparoscopic cholecystectomy. Chirurgia 2016;111(1):26-32. PMID: 10917468. 
12. Flum DR. Intraoperative cholangiography and risk of common bile duct injury during cholecystectomy. JAMA 2003;289(13):1639-1644. DOI: 10.1001/jama.289.13.1639.

13. Urbach DR. Rate of elective cholecystectomy and the incidence of severe gallstone disease. Can Med Assoc J 2005;172(8):1015-1019. DOI: 10.1503/cmaj.1041363.

14. Machi J, Tateishi T, Oishi AJ, et al. Laparoscopic ultrasonography versus operative cholangiography during laparoscopic cholecystectomy: review of the literature and a comparison with open intraoperative ultrasonography. J Am Coll Surg 1999;188(4):360-367. DOI: 10.1016/ s1072-7515(98)00313-5.

15. Jamal K, Smith H, Ratnasingham K, et al. Meta-analysis of the diagnostic accuracy of laparoscopic ultrasonography and intraoperative cholangiography in detection of common bile duct stones. Ann R Coll Surg Engl 2016;98(04):244-249. DOI: 10.1308/rcsann.2016.0068.

16. Aziz O, Ashrafian $\mathrm{H}$, Jones $\mathrm{C}$, et al. Laparoscopic ultrasonography versus intra-operative cholangiogram for the detection of common bile duct stones during laparoscopic cholecystectomy: a metaanalysis of diagnostic accuracy. Int J Surg 2014;12(7):712-719. DOI: 10.1016/j.ijsu.2014.05.038.

17. Li J-W, Feng B, Wu L, et al. Intraoperative cholangiography in combination with laparoscopic ultrasonography for the detection of occult choledocholithiasis. Med Sci Monit 2009;15(9):MT126-MT130. PMID: 19721408

18. Machi J, Oishi AJ, Tajiri T, et al. Routine laparoscopic ultrasound can significantly reduce the need for selective intraoperative cholangiography during cholecystectomy. Surg Endosc 2007;21(2): 270-274. DOI: 10.1007/s00464-005-0817-y.

19. Rhodes M, Nathanson L, O'rourke N, et al. Laparoscopic exploration of the common bile duct: lessons learned from 129 consecutive cases. Br J Surg 1995;82(5):666-668. DOI: 10.1002/bjs.1800820533.
20. Hainsworth PJ, Rhodes M, Gompertz RHK, et al. Imaging of the common bile duct in patients undergoing laparoscopic cholecystectomy. Gut 1994;35(7):991-995. DOI: 10.1136/gut.35.7.991. Available from: http://gut.bmj.com/.

21. Ganguli S, Kruskal JB, Brennan DD, et al. Intraoperative laparoscopic ultrasound, vol. 1. In: Ultrasound clinics. 2006. p. 547-557.

22. Ford JA, Soop M, Du J, et al. Systematic review of intraoperative cholangiography in cholecystectomy. Br J Surg 2012;99(2):160-167. DOI: 10.1002/bjs.7809.

23. Serralta Serra A, Planells Roig M, Bueno Lledo J, et al. The learning curve in ambulatory laparoscopic cholecystectomy. Surg Laparosc Endosc Percutaneous Tech 2002;12(5):320-324. DOI: 10.1097/00129689-200210000-00003.

24. The Southern Surgeons Club, Moore MJ, Bennett CL. The learning curve for laparoscopic cholecystectomy. Am J Surg 1995;170(1):55-59. DOI: 10.1016/s0002-9610(99)80252-9.

25. Polat FR, Abci I, Coskun I, et al. The importance of intraoperative cholangiography during laparoscopic cholecystectomy. JSLS: J Soc Laparoendosc Surg 2000;4(2):103-107. PMID: 10917115; PMCID: PMC3015375

26. Darrien JH, Connor K, Janeczko A, et al. The surgical management of concomitant gallbladder and common bile duct stones. HPB Surg 2015;2015:165068. DOI: 10.1155/2015/165068.

27. Ghazal AH, Sorour MA, El-Riwini M, et al. Single-step treatment of gall bladder and bile duct stones: a combined endoscopiclaparoscopic technique. Int J Surg 2009;7(4):338-346. DOI: 10.1016/ j.ijsu.2009.05.005.

28. Hicken NF, McAllister AJ. Operative cholangiography as an aid in reducing the incidence of "overlooked" common bile duct stones: a study of 1,293 choledocholithotomies. Surgery1964;55(6):753-758. DOI: https://doi.org/10.26611/106837 